\title{
PROSTHETIC PROGRAMME AFTER ABOVE-KNEE AMPUTATION IN CHILDREN WITH SARCOMATA
}

\author{
W. G. COLE, R. W. KLEIN, M. VAN LITH, R. JARVIS
}

From the Royal Children's Hospital and the Central Development Unit, the Department of Veterans' Affairs, Melbourne

\begin{abstract}
A programme for early mobilisation using a temporary prosthesis was evaluated in 17 children who had had an amputation above the knee for sarcomata. The temporary prosthesis had a preformed adjustable polypropylene quadrilateral socket which was able to accommodate changes in the size of the stump during the first few months after amputation. The adjustable sockets were assembled onto wooden knee-shank-foot units or onto modular components covered with foam. The wooden units were better for routine use as more adjustment was possible between the socket and the knee and because they were more durable in active children. Prosthetic fitting usually took one hour and was carried out 10 days after the amputation to coincide with the start of the chemotherapy programme. The prosthesis was cosmetically acceptable, easy to use and provided a simple and economical way of rehabilitating the amputees and restoring their morale.

After two to three months a new prosthesis with a laminated socket suspended by a waistband was supplied. The skin tolerated the closer fit of this socket and the small fluctuations in the size of the stump that occurred with each course of chemotherapy were easily accommodated by varying the thickness of the stump sock. A self-suspending laminated socket was provided after completion of the chemotherapy. The permanent sockets were assembled onto wooden components but the girls usually preferred the modular system covered with foam. The chemotherapy and rehabilitation programmes were successfully co-ordinated so that the children spent as little time as possible away from their normal activities.
\end{abstract}

The most effective method for early rehabilitation of the lower limb amputee is to provide a prosthesis as soon as the wound is healed (McDougall and Emmerson 1977). It is particularly important in children and adolescents with sarcomata treated by amputation above the knee and chemotherapy because of the prolonged and unpleasant nature of the treatment and the uncertain prognosis (Watts 1979).

A temporary prosthesis is usually supplied soon after operation but there is considerable difference of opinion about the type that should be used. The preformed quadrilateral sockets described by McDougall and Emmerson (1977) and Foort (1979) are quick and convenient to use for temporary prostheses but they cannot be adjusted to accommodate the changes in the size of the stump that occur during the first few months after amputation. We used adjustable polypropylene quadrilateral sockets developed by Irons et al. (1977) to accommodate these changes in size. These sockets had previously been used successfully by a group of elderly dysvascular amputees who were unable to use their permanent prostheses because of the fragility of the skin and fluctuations in the size of their stumps. The sockets were found to be light, comfortable and easy to fit and remove. In this paper we report the details of this temporary prosthesis and its use for the early mobilisation of 17 children with sarcomata treated by amputation above the knee and chemotherapy.

\section{PATIENTS}

The temporary prosthesis and our programme for early mobilisation were evaluated in 17 children and adolescents who had had amputations above the knee between March 1978 and March 1981 for sarcomata. There were 10 girls and seven boys, with a mean age of 12.7 years (range 7 to 17 years): 15 had osteosarcomata, one had a Ewing's sarcoma and the remaining patient had a haemangiopericytoma. Those with osteosarcoma were started on a course of adriamycin $(60$ milligrams per square metre) and vincristine (two milligrams per square metre) 10 to 14 days after the amputation. Methotrexate was also given in a randomised trial: 10 children were given moderate doses (200 milligrams per square metre) and five children received high doses ( 7500 milligrams per square metre). Methotrexate and vincristine were given during a three-day admission each month while adriamycin was given at the monthly outpatient visit. A girl aged 15 years with a haemangiopericytoma of the proximal fibula was started on an 18month course of vincristine (1.5 milligrams per square metre), actinomycin $\mathrm{D}$ (1.5 milligrams per square metre) and cyclophosphamide

W. G. Cole, MSc, FRACS, Orthopaedic Surgeon

Department of Orthopaedic Surgery, Royal Children`s Hospital. Flemington Road, Parkville, Victoria 3052, Australia.

R. W. Klein, MB BS. DPRM, Director

$M$. van Lith, BE, MSc(BioEng). Senior Engineer

R. Jarvis. Dip PO. DRS. Senior Prosthetist Orthotist

Central Development Unit. Department of Veterans`Affairs, 131 Sturt Street, South Melbourne, Victoria 3205, Australia.

Requests for reprints should be sent to $\mathrm{Mr}$ W. G. Cole.

(C) 1982 British Editorial Society of Bone and Joint Surgery 0301-620X/82/5112-0586 \$2.00 


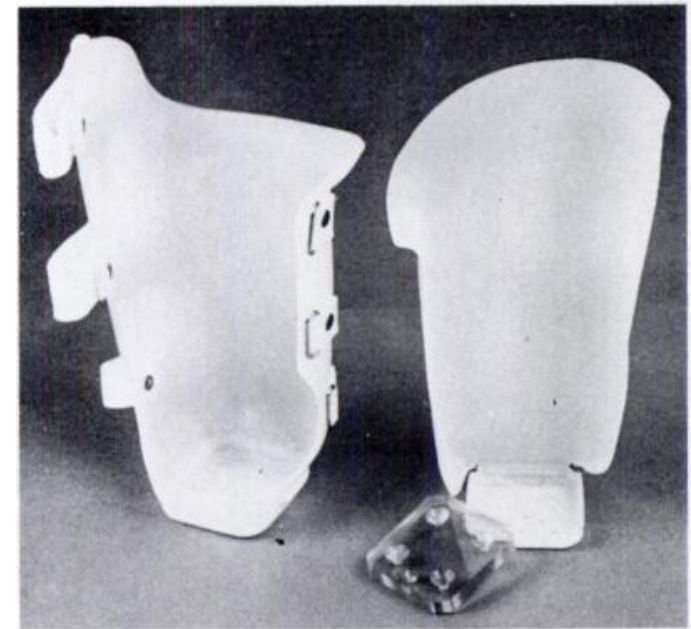

Fig. 1

The adjustable polypropylene quadrilateral socket the two shells are attached distally to the polycarbonate plate and the Velcro fasteners are used to adjust the dimensions of the socket.

(600 milligrams per square metre) 14 days after operation. The remaining patient, a boy aged 10 years with a Ewing's sarcoma of the tibia, had previously received radiotherapy for the primary lesion and had completed an 18 -month course of chemotherapy before having the amputation which was carried out because of fibrosis about the knee induced by the radiation.

Two boys aged 14 and 11 years who had been treated for osteosarcoma died of metastases eight and 16 months after amputation respectively. Two children developed pulmonary metastases while receiving chemotherapy, and another developed pulmonary metastases two-and-a-half years after amputation. There was no evidence of local recurrences or metastases in the other 12 children.

\section{THE PROSTHETIC PROGRAMME}

An important aspect of this programme was the careful preparation of the child and family for the amputation. In an informal meeting before the operation the families were provided with realistic expectations which facilitated early rehabilitation.

After the amputation a crêpe bandage hip spica was applied: the bandage was covered with a stump sock suspended from a belt around the waist. The suction drain was removed two days after the operation but the bandages were otherwise left undisturbed for 10 days. Two days after the operation the patient started walking with crutches and was discharged from hospital on the fourth day to be readmitted on the tenth day for fitting of the temporary prosthesis and to start chemotherapy.

The prosthesis. Preformed polypropylene quadrilateral sockets were obtained from the United States Manufacturing Company (Glendale, California). They are available in right and left sets, 20-centimetre and 28-centimetre lengths and in four inlet circumferences-small (42 centimetres). medium ( 45 centimetres), large ( 50 centimetres) and very large (54 centimetres). The sockets are split in the frontal plane and the two shells are joined distally to a polycarbonate plate (Fig. 1). The socket has three straps with Velcro fasteners located horizontally across the front of the socket which can be adjusted to accommodate changes in the size of the stump. Suspension is provided by a waistbelt or a shoulder-strap attached to the socket.

The socket can be attached directly to a modular knee-shank-foot unit obtained from the United States Manufacturing Company. This modular unit consists of a single-axis knee joint attached to an aluminium alloy tubular shank and a SACH (solid-ankle-cushion-heel) foot. The socket can also be easily attached to a North-Western intermittent friction knee-shank unit with a SACH foot. The

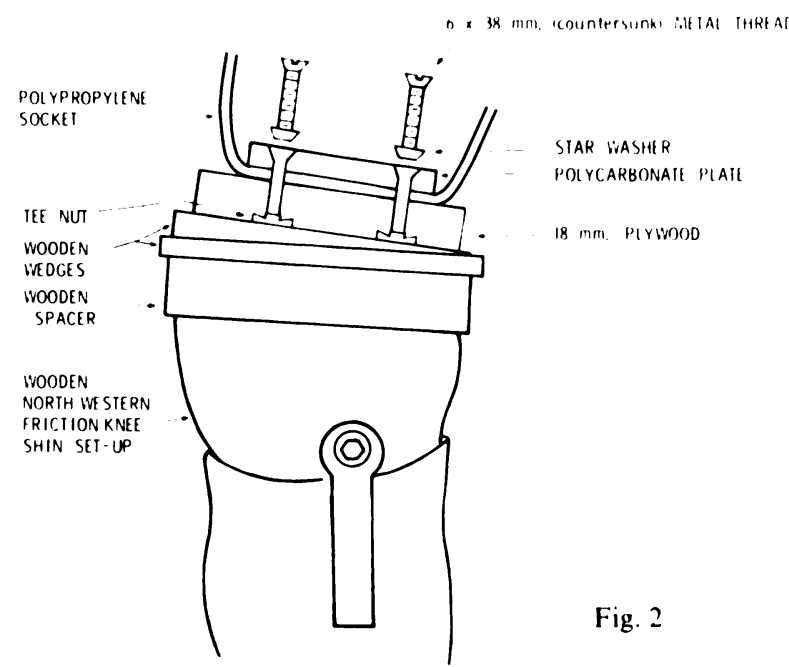

Lateral diagram showing the method of attachment of the polypropylene socket to the wooden knee

manufacturer's screws and nuts were removed from the polycarbonate plate of the socket and were replaced with metal threads that were inserted through the holes into nuts embedded into a piece of 18 millimetre plywood. Pre-cut wooden blocks and wedges were inserted between the plywood and the knee to give the desired length and correct alignment (Fig. 2).

Fitting and alignment. The prosthesis could be assembled and bench aligned before the child attended for the first fitting provided the appropriate measurements had been given to the prosthetist. Ten days after the operation the prosthesis was fitted and dynamically aligned. This could be done in one hour if the preliminary assembly and alignment had already been done. The fit of the socket was checked after a 5-ply woollen sock had been drawn over the stump. The child started standing and walking immediately and later on the same day started the first course of cheinotherapy. During the three-day admissions for chemotherapy, the wooden inserts between the socket and the knee of the prosthesis were covered with polyester resin to match the resin already on the shank (Fig. 3). During this time the modular units were covered with cosmetic foam which was shaped to match the dimensions of the normal leg.

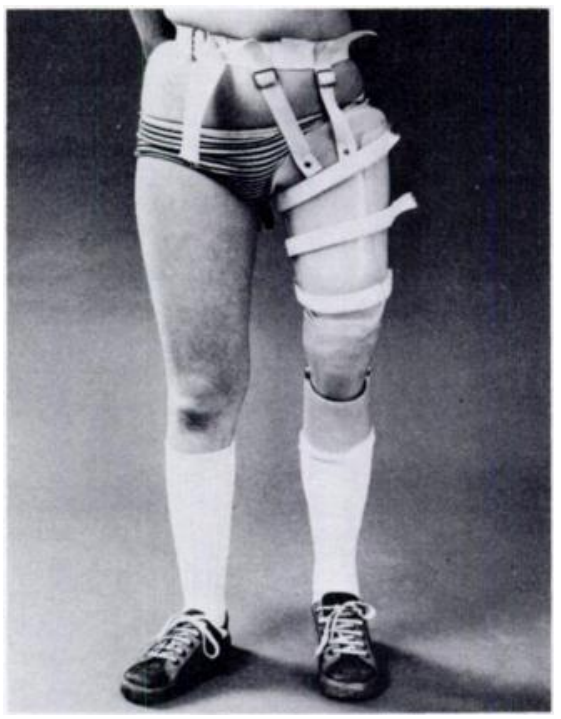

Fig. 3

A girl aged 11 years wearing a completed prosthesis. The adjustable polypropylene socket has been assembled onto a wooden knee-shank-SACH foot unit. The wooden wedges and spacers have been covered with polyester resin. 
Later prosthetic care. The socket was checked and adjusted by the prosthetist during the child's monthly admissions for chemotherapy; a physiotherapist also checked the adequacy of the bandaging of the stump and supervised exercises to strengthen the stump and trained the patient in the use of the prosthesis.

When the size of the stump was stable a cast was taken for a new prosthesis consisting of a laminated quadrilateral socket suspended from the waist, and either a wooden North-Western knee-shank-foot unit or an Otto Bock modular knee-shank-foot unit covered with foam. A self-suspending total contact socket with a valve was supplied after the child had completed the full course of chemotherapy.

\section{RESULTS}

Stump maturation. Three patterns of stump maturation were observed. In nine children the initially swollen stump rapidly decreased in size over the first two to three months and only small monthly variations were noted during the remainder of their chemotherapy. However, larger monthly variations in the size of the stump were observed in the five children receiving high doses of methotrexate. The third pattern of maturation was observed in three children in whom the size of the stump progressively increased as the child's overall weight increased. As this was due to overeating, all of the children were reviewed regularly during each admission by our dietitian.

Socket fit and adjustment. In 14 children the initial fit of the socket was satisfactory. In each child there was additional space in the socket below the end of the stump, and although Irons et al. (1977) recommended that the additional space should be filled with foam we did not find it necessary.

We were able to alter the circumference of the inlet by three to five centimetres with the proximal strap to accommodate changes in the size of the stump. However, only a negligible amount of adjustment was possible with the middle strap and none at all with the distal strap. As a consequence, the distal half of the stump was usually poorly supported by the end of the second month after the amputation.

In three children the preformed adjustable sockets did not fit satisfactorily. The stump of the youngest patient, a girl aged seven years, was too small for even the smallest socket while the largest sockets were found to be too small for two girls aged 14 years who had had amputations at the junction of the proximal and middle thirds of the femur. A modified polypropylene socket was used for the seven-year-old girl while plaster sockets and laminated sockets were used for the two older girls; these sockets required more of the prosthetist's time to fit and were less convenient for the children. As the size of the sockets could not be adjusted they had to be changed on several occasions during the first three months after amputation.

Knee-shank-foot units. The standard wooden knee-shank and $\mathrm{SACH}$ foot units obtained from reclaimed permanent above-knee prostheses were ideal as they could be easily assembled and aligned and they were able to withstand the recreational and sporting activities of this age group. However, the modular system was not considered to be suitable for routine use in children as the cosmetic foam covers were not sufficiently durable although they were suitable for less active teenage girls. We did not supply the modular system without the cosmetic cover as all of the children, regardless of their age and sex, wished to have a prosthesis which matched the appearance of their normal leg.

The limited amount of adjustment that was possible between the socket and the modular unit made it difficult to align the prosthesis correctly in some children. However, the socket could be easily aligned on the wooden units and adjusted by inserting extra wedges. Although the adjustable socket could be attached more rapidly to the modular unit than to the wooden unit extra time was required to fit and shape the cosmetic foam cover.

Gait. All of the children rapidly learnt to stand and walk and they were sufficiently confident to return to school within a few weeks of receiving their prosthesis. It was important that the assessment of the fit of the socket and gait, as well as training in the use of the prosthesis, were carried out when the child was feeling well. As a consequence these assessments were usually made during the first day of each admission before the administration of the chemotherapeutic agents.

Careful bandaging, exercises to strengthen the stump and a well-fitting socket were considered to be important factors in achieving a good gait. However, it was noted that the children receiving chemotherapy were weaker and had less endurance than other children who had had amputations above the knee without chemotherapy. Although there were no objective clinical signs of peripheral neuropathy in the other leg it was likely that the chemotherapy had resulted in muscular weakness.

Cosmetic appearance. All of the children considered their appearance to be as important as their ability to walk. The children were pleased with their temporary prosthesis and with their own appearance when fully dressed. It was clear that the children wished to have a prosthesis that resembled, as closely as possible, the permanent prosthesis. They would not accept a socket attached to an above-knee pylon although this system is commonly used in the early rehabilitation of adult amputees.

Later prosthetic care. A new prosthesis was ordered during the second to third month after the amputation as the adjustable socket was usually unable to provide adequate support for the stump beyond this time. However, a smaller adjustable socket was not used in the new prosthesis as the fluctuations in the size of the stump observed after chemotherapy were not great enough even in those patients given high doses of methotrexate. The new prosthesis containing a laminated quadrilateral socket suspended by a waistbelt was cosmetically more acceptable to the children and provided good support of 
the stump and enabled them to walk well. Most children continued to wear a stump sock with this prosthesis. The skin was protected by the sock and the fluctuations in the size of the stump were readily accommodated by varying the thickness of the sock. Most children required two of these prostheses during their 18-month course of chemotherapy.

The self-suspending total contact laminated sockets with a valve were not successfully used while the children were still receiving chemotherapy. Two girls requested new prostheses of this type three months after their amputation but they developed severe skin rashes over the stump and they were frequently unable to fit their prosthesis because of fluctuations in the size of the stump. However, these problems were usually not encountered after the course of chemotherapy had been completed.

The laminated sockets were usually assembled onto North-Western wooden knee-shank units. However, most of the teenage girls were provided with the Otto Bock modular system covered with foam.

\section{DISCUSSION}

The temporary prosthesis and the programme for early mobilisation used in this study were shown to be simple and effective. The preformed adjustable polypropylene sockets could be rapidly assembled onto either of the two knee-shank-foot units used in the programme and as a result the children received their prostheses 10 days after operation with a minimal amount of inconvenience to the child and the prosthetist. Successful fitting improved the morale of the children and enabled them to return to school and to many of their previous activities within a few weeks. Early fitting of the prosthesis and the regular reviews also helped to maintain the family's confidence in the treatment and helped the children to cope with their chemotherapy programme.

The prosthetic and chemotherapy programmes were successfully co-ordinated so that most of the prosthetic procedures were carried out during the attendances for chemotherapy. This arrangement dramatically reduced the number of visits to hospital that would otherwise have been necessary. The use of the adjustable socket in our temporary prosthesis also removed the need for frequent socket changes that would have been required had we used the non-adjustable sockets described by McDougall and Emmerson (1977) and Foort (1979). The availability of sockets in a range of sizes enabled the prosthesis to be fitted within one hour and removed the need to cast the painful stump of the recent amputee. The sockets were also comfortable, easy to fit and remove and could be re-used by other children.

Other preformed adjustable polypropylene sockets are available but they were not evaluated in this study. The socket provided by Blatchford as part of their lightweight modular above-knee prosthesis is similar in appearance to the socket used in our study. A polypropylene socket shaped like a tulip with medial and lateral adjustment straps was recommended by Watts (1979) for use in children with sarcomata.

An alternative method of providing temporary sockets is required for children whose stumps are either too small or too large for the available preformed adjustable quadrilateral sockets. One alternative is to make a vacuum formed polypropylene socket from a plaster cast of the stump. Another alternative would be to use the light-weight, rigid polyurethane foam socket which is formed directly to the patient's stump while the polyurethane hardens (Holter et al. 1980). In either case the sockets would need to be changed during the first few months after amputation.

We agree with Watts (1979) that the fragility of the skin and fluctuations in the size of the stump that occur while the children are receiving chemotherapy make it difficult for them to use a self-suspending total contact socket. However, we did not consider that it was necessary for the children to use the adjustable socket for more than two to three months since after this time they were able to use a laminated socket with a stump sock and waist suspension. The laminated socket provided better support for the stump and was cosmetically more attractive than the adjustable socket.

In our study the North-Western wooden knee-shankfoot units were found to be most useful for routine use in the temporary and permanent prostheses. The reclaimed components used in the temporary prostheses were readily available and therefore reduced the cost.

The temporary prosthesis used in our study can also be used to facilitate the early rehabilitation of children having above-knee amputations for non-malignant diseases. The preformed adjustable socket was also used as a permanent socket in children with the KlippelTrenauney-Weber syndrome when they were unable to wear a standard prosthesis because of marked fluctuations in the size of the stump.

\section{REFERENCES}

Foort J. Socket design for the above-knee amputee. Prosthet Orthot Int 1979:3:73-81.

Holter W, Echterhof M, Blömer A, Verfürdin H. The management of amputations of the leg using a new rigid foam plaster for prosthetic fitting. Int Orthop 1980:4:73-7.

Irons GB, Mooney V, Putnam S, Quigley M. A lightweight above-knee prosthesis with an adjustable socket. Orthot Prosthet $1977: 31: 3-15$. McDougall A, Emmerson A. The preformed socket and modular assembly for primary amputees. J Bone Joint Surg [Br] 1977:59-B:77-9.

Watts HG. Surgical management of malignant bone tumours in children. In: Jaffe N, ed. Bone Tumors in Children. Massachusetts: PSG Publishing Company, 1979:131-46. 